\title{
Gerência do cuidado de enfermagem no serviço de métodos endoscópicos gástricos: relato de experiência
}

\author{
Nursing care management in the servisse of gastric endoscopic methods: experience report \\ La gestión del cuidado de enfermería al servicio de los métodos endoscópicos gástricos: informe de \\ experiencia
}

Recebido: 05/09/2021 | Revisado: 11/09/2021 | Aceito: 16/09/2021 | Publicado: 17/09/2021

Sônia Cristina Chagas Peçanha

ORCID: https://orcid.org/0000-0001-8808-2976 Universidade Federal Fluminense, Brasil E-mail: sccpecanha@id.uff.br

Barbara Pompeu Christovam

ORCID: https://orcid.org/0000-0002-9135-8379 Universidade Federal Fluminense, Brasil E-mail: barbarachristovam@id.uff.br

Lucas Rodrigo Garcia de Mello

ORCID: https://orcid.org/0000-0002-4833-606X Universidade Federal Fluminense, Brasil

E-mail: lucasgmello@ig.com.br

Érica Brandão de Moraes

ORCID: https://orcid.org/0000-0003-3052-158X Universidade Federal Fluminense, Brasil

E-mail: ericabrandao@id.uff.br

Cláudio José de Souza

ORCID: https://orcid.org/0000-0001-7866-039X Universidade Federal Fluminense, Brasil

E-mail: claudioenfo@gmail.com

\begin{abstract}
Resumo
Objetivo: relatar a experiência de enfermeiros na gerência do cuidado ao paciente no serviço de métodos endoscópicos gástricos. Método: trata-se de um relato de experiência da vivência profissional de um grupo de enfermeiros no Serviço de Métodos Endoscópicos Gástricos, em um hospital do Rio de Janeiro, em março 2020-21. Resultados: a vivência profissional no período de pandemia possibilitou a reflexão sobre a prática do enfermeiro na gerência das ações de cuidados havendo, nas ações diretas, a implementação de protocolos, como o de triagem do paciente, e ajustes em outros, como, o de recuperação pós-procedimento; e nas ações indiretas, cálculos sobre previsão e provisão de materiais e equipamentos, modificações nos fluxos de trabalho, fixação de sinalizações e reforços teórico-práticos nas rotinas de desinfecção e limpeza. Considerações finais: a experiência permitiu o desenvolvimento de um olhar crítico e reflexivo acerca das ações gerenciais e das medidas de biossegurança, em vigência de pandemia, favorecendo o raciocínio clínico e o aprimoramento das ações diretas e indiretas contemplando paciente, família/acompanhante, profissionais e instituição.
\end{abstract}

Palavras-chave: Cuidados de enfermagem; Infecções por coronavírus; Administração dos cuidados ao paciente; Assistência ao paciente; Organização e administração.

\begin{abstract}
Objective: to report the experience of nurses in patient care management in the service of gastric endoscopic methods. Method: this is an experience report of the professional experience of a group of nurses in the Gastric Endoscopic Methods Service, in a hospital in Rio de Janeiro, in March 2020-21. Results: the professional experience in the pandemic period allowed for reflection on the practice of nurses in the management of care actions, with direct actions involving the implementation of protocols, such as patient screening, and adjustments in others, such as postprocedure recovery; and in indirect actions, calculations on forecasting and provision of materials and equipment, changes in work flows, setting of signs and theoretical-practical reinforcements in disinfection and cleaning routines. Final considerations: the experience allowed the development of a critical and reflective look at managerial actions and biosafety measures, in the presence of a pandemic, favoring clinical reasoning and the improvement of direct and indirect actions contemplating the patient, family/companion, professionals and institution.
\end{abstract}

Keywords: Nursing care; Coronavirus infections; Administration of patient care; Patient care; Organization and administration. 


\begin{abstract}
Resumen
Objetivo: reportar la experiencia del enfermero en la gestión de la atención al paciente al servicio de los métodos endoscópicos gástricos. Método: se trata de un relato vivencial de la experiencia profesional de un grupo de enfermeras del Servicio de Métodos Endoscópicos Gástricos, en un hospital de Río de Janeiro, en marzo 2020-21. Resultados: la experiencia profesional en el período pandémico permitió reflexionar sobre la práctica del enfermero en la gestión de las acciones de atención, con acciones directas que involucran la implementación de protocolos, como el cribado de pacientes, y ajustes en otros, como la recuperación posprocedimiento; y en acciones indirectas, cálculos de previsión y dotación de materiales y equipos, cambios en los flujos de trabajo, colocación de señalización y refuerzos teórico-prácticos en las rutinas de desinfección y limpieza. Consideraciones finales: la experiencia permitió desarrollar una mirada crítica y reflexiva sobre las acciones gerenciales y medidas de bioseguridad, ante la presencia de una pandemia, favoreciendo el razonamiento clínico y la mejora de las acciones directas e indirectas contemplando al paciente, familia / acompañante, profesionales e institución.
\end{abstract}

Palabras clave: Atención de enfermería; Infecciones por coronavirus; Administración de la atención al paciente; Atencion al paciente; Organización y administración.

\title{
1. Introdução
}

Os coronavírus são vírus de ácido ribonucleico que podem causar doenças respiratórias, entéricas, hepáticas e neurológicas em diferentes espécies animais, incluindo camelos, bovinos, gatos e morcegos. Até o momento, foram identificados sete tipos de coronavírus capazes de infectar humanos, sendo o último, o SARS-CoV-2 (Brasil, 2021; Cascella, et al., 2020). A doença foi chamada de Covid-19, uma sigla que vem do inglês Coronavirus Disease, do ano de 2019 (Who, 2020a). Os primeiros casos desta doença foram identificados em um grupo de pessoas, em um mercado de frutos do mar e animais vivos na cidade de Wuhan, a maior área metropolitana da província de Hubei, na China (Brasil, 2021; Opas, 2021a; Cascella, et al., 2020; Hassan, et al., 2020).

Devido a fácil propagação, a disseminação do vírus para o resto do mundo se deu de maneira rápida nos meses seguintes, principalmente pela facilidade dos mais variados tipos de transeuntes em diversos países do globo terrestre. Ademais, pode-se considerar como um agente contribuidor de disseminação, uma parcela dos indivíduos infectados não apresentar os sintomas clássicos da doença, estando considerados no hall dos sintomas leves manifestando febre, tosse seca e cansaço (Opas, 2021a; Who, 2020b). Uma minoria, porém, significativa, devido ao grande número de infectados e possíveis comorbidades associadas, evoluem com sintomas graves necessitando de internação hospitalar, internação em terapia intensiva e havendo a possibilidade de um desfecho desfavorável, como o óbito (Brasil, 2021; Cascella, et al., 2020; Hassan, et al., 2020).

Sabe-se que a transmissão do vírus ocorre de pessoa para pessoa pelo ar (contato pessoal próximo) ou por contato com objetos ou superfícies contaminadas, seguido de contato com a boca, nariz ou olhos. Medidas de biossegurança são disseminadas a fim de prevenir a propagação do vírus, sendo a higienização das mãos e respiratória as medidas mais recomendadas pelos especialistas da área (Cascella, et al., 2020; Hassan, et al., 2020). Neste contexto, as organizações de saúde coordenam fluxos de informações e emitem diretrizes e recomendações para melhor mitigar o impacto da doença (Brasil, 2020; Opas, 2021b; Who, 2020c. Ao mesmo tempo, cientistas de todo o mundo desenvolvem estudos com informações atualizadas sobre os mecanismos de transmissão, o espectro clínico da doença, novos diagnósticos e estratégias de prevenção e terapêutica (Cascella et al., 2020; Hassan, et al., 2020). Em agosto de 2021, havia mais de 290 vacinas candidatas em desenvolvimento incluindo-se, mais de 100 que já estão sendo testadas em ensaios clínicos (Opas, 2021c).

O impacto da pandemia no mundo promoveu rápidas mudanças no cuidado prestado a qualquer tipo de paciente. No cenário hospitalar, medidas de prevenção e controle de infecção foram implementadas pelos profissionais para evitar ou reduzir ao máximo a transmissão de microrganismos durante a assistência à saúde realizada, incluindo, nos casos suspeitos ou confirmados de infecção pelo SARS-CoV-2, segundo as evidências disponíveis, em todas as etapas do atendimento de saúde, 
desde sua chegada, triagem, espera, durante toda a assistência prestada, até sua a sua alta, transferência ou óbito (Brasil, 2021; Who, 2021a).

Neste sentido, destaca-se a relevância da Enfermagem na deteç̧ão e avaliação dos casos suspeitos, não apenas em razão de sua capacidade técnica, mas também por constituírem-se no maior número de profissionais da área da saúde, e serem da única categoria profissional que está nas 24 horas junto ao paciente. A pluralidade da formação do enfermeiro e sua posição de liderança na equipe, coloca o profissional de enfermagem como protagonista para evitar a transmissão sustentada no território nacional (Cofen, 2020a). Assim, seguindo recomendações gerais para organização dos serviços de saúde e preparo das equipes de enfermagem, o enfermeiro adotou medidas para adequar as mudanças necessárias ao enfrentamento da pandemia da COVID-19 (Cofen, 2020b).

A prática da gerência do cuidado de enfermagem realizada pelos enfermeiros nas unidades assistenciais, consiste numa sistematização de atividades que envolve diferentes níveis de complexidade para o planejamento e para a organização do cuidado de enfermagem, do processo de trabalho da equipe de enfermagem, do ambiente terapêutico e, dos recursos materiais e dos equipamentos necessários à implementação de ações de caráter instrumental e expressivo na prestação do cuidado direto e indireto aos pacientes do setor (Christovam, Porto \& Oliveira, 2012; Santos \& Lima, 2011; Joaquim \& Silvino, 2020).

Compreende-se que a gerência do cuidado de enfermagem seja uma ação transversal a qualquer tipo de assistência e que envolve ações de cuidado direto e indireto aos pacientes, de carácter instrumental e principalmente expressivo, fazendo com que os profissionais possam em seu cotidiano mobilizar habilidades cognitivas, analíticas e comportamentais atuando nos diversos setores hospitalares com o propósito de atender as necessidades básicas dos pacientes e ao mesmo tempo, as necessidades dos familiares/acompanhantes, equipe e instituição (Christovam, Porto \& Oliveira, 2012; Gomes, et al., 2021), de modo a zelar pela qualidade do cuidado, garantindo os objetivos de biossegurança sejam contemplados por estas ações pois, é um período de pandemia por coronavírus (Lima, et al., 2020).

Postos os fatos, o presente estudo tem como objetivo, relatar a experiência de enfermeiros na gerência do cuidado ao paciente no serviço de métodos endoscópicos gástricos.

\section{Metodologia}

Trata-se de um relato de experiência, de caráter descritivo e qualitativo acerca da gerência do cuidado a paciente no Serviço de Métodos Endoscópicos Gástricos (SMEG), sob a ótica da enfermagem. Deste modo, este estudo descreve e analisa particularidades de uma experiência com dados e informações de modo a contribuir com o saber na área de conhecimentos na qual for vivenciada (Pereira, et al., 2018). A ideia de socializar este conhecimento que se deu a partir das reuniões do serviço, observações, estudos e discussões entre os profissionais de enfermagem da instituição, visto que, a priori esta unidade não se encontrava preparada para receber paciente em vigência de pandemia por coronavírus. $O$ estudo foi formalizado a partir da vivência de um grupo de enfermeiros lotados no SMEG de um hospital universitário, localizado no município do Rio de Janeiro, onde são realizados diversos tipos de exames endoscópicos por via alta e baixa com fins diagnósticos e terapêuticos, no período de março de 2020 a março de 2021.

Com base nas discussões feitas por estes profissionais, este grupo procurou contextualizar estas experiências diárias da gerência do cuidado em um relato de experiência, compilando neste artigo o que os autores compreendem com eixo norteador para que a gerência do cuidado se efetive. Assim, além da contextualização da unidade de trabalhos, os autores procuraram estabelecer o que eles compreendem como cuidado direto e indireto.

A equipe de enfermagem com expertise para atuar no setor é composta por 1 profissional diarista, enfermeiro; e 24 plantonistas, sendo 4 enfermeiros e 20 técnicos / auxiliares de enfermagem. Os plantonistas trabalham das 07 às 19 horas, escala 12 x 36 , de segunda a sexta-feira. 
O cenário do estudo é um hospital universitário, localizado no Rio de Janeiro, e possui 9 salas para exames diagnósticos e procedimentos, sendo os mais frequentes: endoscopia digestiva alta, colonoscopia, colangiopancreatografia endoscópica retrógrada (CPRE), entre outros.

Como trata-se de um relato, não houve a necessidade de encaminhamento ao comitê de ética, uma vez que não se trata de um estudo com seres humanos.

\section{Resultados e Discussão}

$\mathrm{O}$ processo de gerenciamento do cuidado aos pacientes se deu de maneira ampla e multiprofissional com a implantação de práticas assistenciais, embasado nas recomendações da American Society for Gastrointestinal Endoscopy (Asge, 2021), Sociedade Brasileira de Endoscopia Digestiva (Sobed, 2020), do Ministério da Saúde e da Agência Nacional de Vigilância Sanitária (Brasil, 2021), do nosso município (Prefeitura do Rio de Janeiro, 2021), conforme as informações sobre a doença foram sendo atualizadas (Opas, 2021a).

De acordo com o conceito Christovam, Porto e Oliveira, (2012), a gerência do cuidado, envolve ações de cuidado direto e indireto aos pacientes, dessa forma, serão descritos as ações de acordo com essa perspectiva, conforme Quadro 1.

Quadro 1 - Ações de cuidado direto e indireto que envolvem a gerência do cuidado aos pacientes no serviço de métodos endoscópicos gástricos.

\begin{tabular}{|l|l|}
\hline TIPOS DE AÇÕES & \multicolumn{1}{c|}{ GESTÃO DO CUIDADO } \\
\hline \multirow{5}{*}{ AÇÕES INDIRETAS } & Previsão de materiais para a realização procedimentos; \\
\cline { 2 - 2 } & Previsão, provisão de equipamento de proteção individual (EPI); \\
\cline { 2 - 2 } & Treinamento sobre paramentação e desparamentação dos EPIs; \\
\cline { 2 - 3 } & Sinalização para adoção de medidas distanciamento social; \\
\cline { 2 - 3 } & Ajuste no fluxo de limpeza e desinfecção dos materiais; \\
\cline { 2 - 3 } & Atualização das rotinas de desinfecção e limpeza das salas de procedimentos; \\
\cline { 2 - 3 } & Adequação do quantitativo de dispensers de álcool a 70\%; \\
\cline { 2 - 3 } AÇÕES DIRETAS & Triagem e encaminhamento de profissionais com estado gripal para o setor de medicina do trabalho. \\
\cline { 2 - 3 } & Implementação de protocolo de triagem no acolhimento do paciente; \\
\cline { 2 - 3 } & Ajuste no protocolo de recuperação pós-exame; \\
\cline { 2 - 3 } & Triagem do familiar/acompanhante; \\
\cline { 2 - 3 } & Orientação sobre as medidas de prevenção e detecção precoce dos sinais e sintomas. \\
\hline
\end{tabular}

Fonte: Autores (2021).

Verificou-se que na prática da gerência do cuidado, em virtude da pandemia por coronavírus, houve necessidade de se rever o fluxo de atendimento conferindo segurança para os pacientes, acompanhantes e profissionais. A sistematização das atividades envolveu diferentes níveis de complexidade assistencial pois o paciente chega ao serviço deambulando (salvo em situações especiais), acompanhado, é sedado para a realização do procedimento e após o despertar anestésico, é liberado.

As ações do cuidado indireto exigiram uma dedicação integral para estabelecer todas as medidas necessárias para garantia da segurança durante toda a permanência no serviço. Destaca-se a preocupação com previsão e provisão dos materiais, visto que o recurso poderia ser afetado devido escassez de matéria prima. Ademais, a inclusão de novos Equipamentos de Proteção Individual (EPIs) no cotidiano dos profissionais, sobretudo, na capacitação da paramentação e desparamentação de forma segura. 
Os procedimentos endoscópicos apresentam alto risco de contaminação para a equipe multiprofissional, pacientes e acompanhantes no setor, devido ao processo de aerossolização que ocorre durante o exame e uso do equipamento. Sendo assim, somando este fato ao conhecimento de que grande parte da transmissão do SARS-CoV-2 se dá através de portadores assintomáticos, oligossintomáticos e não diagnosticados, foram criadas estratégias para minimizar os riscos durante o enfrentamento da pandemia, para evitar a contaminação e disseminação do coronavírus.

A sinalização para adoção de medidas de distanciamento social, realizadas através da fixação de cartazes, orientando o quantitativo ideal de pessoas concomitantemente nos espaços, foi extremamente necessária para delimitar os comuns do setor e reforçar a importância para prevenção da doença.

Outro ponto importante foi o reforço do uso obrigatório de EPI na limpeza mecânica do equipamento com treinamento e realização de reuniões com a equipe de enfermagem robustecendo a importância de seguir rigorosamente a política de reprocessamento do endoscópio como um método seguro e eficiente para evitar a propagação da infecção viral; além de orientar-se quanto à realização do teste de infiltração, apenas antes dos exames.

Com o intuito de atender as exigências tanto dos profissionais responsáveis pela Comissão de Controle de Infecção Hospitalar (CCIH), quanto pelas normativas do Ministério da Saúde, a unidade se preocupou em obedecer aos critérios de distanciamento social. Deste modo, os exames foram agendados com uma margem de segurança para a realização entre um e outro, havendo tempo hábil para realização do procedimento, limpeza da sala e liberação de um paciente antes da entrada do próximo.

Já sobre as ações de cuidado direto, destacam-se alterações importantes na avaliação inicial do paciente, com o estabelecimento de um protocolo de triagem no acolhimento do paciente para estratificar o risco de Covid-19, incluindo a investigação sobre a presença de sintomas gripais nos últimos 14 dias, ou ainda, anosmia ou perda do paladar. Foram incluídos: a aferição da temperatura corporal e saturação de oxigênio $\left(\mathrm{SPO}_{2}\right)$, além do termo de consentimento do procedimento informando sobre os riscos do exame.

É importante ressaltar que, inicialmente entre abril e junho de 2020, todos os exames e procedimentos endoscópicos eletivos, foram suspensos; somente os exames de endoscopia de emergência e urgência como, por exemplo, hemorragias digestivas, colangites, impactação de corpo estranho foram mantidos.

Neste contexto, emergiu a necessidade de triagem não apenas do paciente, mas também do familiar/acompanhante. Caso qualquer um desses apresentasse uma temperatura igual ou superior a $37.5^{\circ} \mathrm{C}$, o exame era reagendado. Na sala de recuperação pós-exame, além do distanciamento social, limitou-se a presença de acompanhantes apenas nos casos previstos em leis. Afora estes cuidados, também foi oferecido álcool em gel 70\%, máscara cirúrgica (em caso de estar danificada) e a orientação de permanecer com a mesma durante todo tempo, sendo retirada apenas na hora do exame e recolocada imediatamente após o término.

Neste cenário, a experiência encontrou certo grau de dificuldade relacionada com as recomendações que norteiam os cuidados, pois, levando-se em consideração o fato de que a Covid-19 é uma doença recentemente descoberta e que muitas informações têm sido agregadas no decorrer dos dias devido à quantidade de estudos em andamento, várias atualizações foram publicadas no período, havendo a necessidade de os enfermeiros acompanharem as novas publicações e adequar as ações de cuidado. Por outro lado, estas mesmas atualizações facilitaram na implementação de ações de cuidados condizentes a situação de pandemia além de conferir segurança à equipe ao desenvolver seu trabalho combatendo o coronavírus.

\section{Considerações Finais}

A partir da vivência profissional de enfermeiros na gerência do cuidado ao paciente no serviço de métodos endoscópicos gástricos, constatou-se que na pandemia por coronavírus a experiência permitiu o desenvolvimento de um olhar 
crítico e reflexivo acerca das ações gerenciais havendo planejamento, organização, coordenação e avaliação das ações e das medidas de biossegurança, em vigência de pandemia de Covid-19, favorecendo o raciocínio clínico e o aprimoramento das ações contemplando paciente, família/acompanhante, profissionais e instituição.

Enfatiza-se a relevância das atualizações científicas sendo primordial para a capacitação dos profissionais, assegurando a qualidade e a segurança na assistência prestada, além de destacar-se a necessidade da realização de novos estudos com a quantificação de indicadores assistenciais para avaliar a assistência e as atividades do serviço conferindo maior visibilidade à atuação dos enfermeiros.

Quanto às sugestões para trabalhos futuros, espera que as novas pesquisas fomentem reflexões e discussões quanto aos cuidados diretos, na qual consiste nas orientações dadas ao paciente que fará o procedimento endoscópico, isto é, antes, durante e após procedimento realizado procurando elucidar todas as dúvidas bem como, amenizar os possíveis medos e anseios. No que tange aos cuidados indiretos, espera-se que os profissionais atuantes possam estar realizando avaliações periódicas quanto aos riscos ocupacionais que o ambiente oferece aos profissionais de saúde, bem como, o pessoal do serviço de apoio orientando quanto a rotina de limpeza e a desinfecção terminal dos equipamentos e dos ambientes conforme normas preconizadas pela Comissão de Controle de Infecção Hospitalar local. Deste modo, espera-se que este conhecimento adquirido nesta unidade possa ser aplicável as várias áreas assistenciais da unidade promovendo qualidade e segurança para o paciente e para a equipe multiprofissional.

\section{Referências}

American Society for Gastrointestinal Endoscopy. (2021). Key resources: ASGE Guidelines. https://www.asge.org/home/resources/key-resources/guidelines.

Brasil. Ministério da Saúde. (2020). Plano de Contingência Nacional para Infecção Humana pelo novo Coronavírus COVID-19. COE/SVS/MS. Brasília/DF. https://portalarquivos2.saude.gov.br/images/pdf/2020/fevereiro/13/plano-contingencia-coronavirus-COVID19.pdf.

Brasil. Agência Nacional de Vigilância Sanitária. (2021). Nota Técnica GVIMS/GGTES/ANVISA No 04/2020. (2020). Orientações para serviços de saúde: Medidas de prevenção e controle que devem ser adotadas durante a assistência aos casos suspeitos ou confirmados de infecção pelo novo coronavírus (SARSCoV-2) - atualizada em 25/02/2021. https://www.gov.br/anvisa/pt-br/centraisdeconteudo/publicacoes/servicosdesaude/notas-tecnicas/nota-tecnicagvims_ggtes_anvisa-04_2020-25-02-para-o-site.pdf.

Cascella, M., Rajnik, M., Cuomo, A., Dulebohn, S. C., \& Di Napoli, R. (2020). Features, evaluation and treatment coronavirus (COVID-19). In Statpearls. StatPearls Publishing. https://www.ncbi.nlm.nih.gov/books/NBK55477/6.

Christovam, B. P., Porto, I. S. \& Oliveira, D. C. (2012). Gerência do cuidado de enfermagem em cenários hospitalares: a construção de um conceito. Rev. esc. enferm., 46(3), 734-41. https://doi.org/10.1590/S0080-62342012000300028.

Conselho Federal de Enfermagem. (2020a). Recomendações gerais para organização dos serviços de saúde e preparo das equipes de enfermagem: as unidades de saúde devem se adequar às mudanças necessárias para enfrentamento da pandemia da COVID-19. http://www.cofen.gov.br/wpcontent/uploads/2020/04/cofen_covid-19_cartilha_v3-4.pdf

Conselho Federal de Enfermagem. (2020b). Nota técnica ${ }^{\circ}$ 01/2020. Orientações sobre o novo coronavírus (COVID-19). Brasília: DF. http://www.cofen.gov.br/cofen-publica-nota-tecnica-sobre-o-coronavirus_77070.html.

Gomes, R., Belarmino, A. C., Pinho, C. M., Bastos, P. O., Cavalcante, A. A. N., \& Alves, L. C. (2021). Leadership in hospital nursing: Perception of managing nurses. Research, Society and Development, 10(9), e35410917890. https://doi.org/10.33448/rsd-v10i9.17890

Hassan, S. A., Sheikh, F. N., Jamal, S., Ezeh, J. K., \& Akhtar, A. (2020). Coronavirus (COVID-19): A Review of Clinical Features, Diagnosis and Treatment. Cureus, 12(3), e7355.

Joaquim, F. L., \& Silvino, Z. R. (2020). Validation of management technology for the care of patients with chronic venous ulcers, treated at outpatient clinics in the hospital context. Research, Society and Development, 9(8), e103985256. https://doi.org/10.33448/rsd-v9i8.5256

Lima, L. S. C., Soares, S. S. S., Carvalho, E. C., Varella, T. C. M. y M. L., Santos, D. M., Silva, P. A. S., \& Souza, N. V. D. O. (2020). Reflexões sobre biossegurança no contexto da COVID-19: repercussões para profissionais e para população. Research, Society and Development, 9(9), e818997993. https://doi.org/10.33448/rsd-v9i9.7993

Organização Pan-Americana de Saúde. Organização Mundial da Saúde (2021a). Folha informativa sobre COVID-19. https://www.paho.org/pt/covid19\#: :text=Os\%20sintomas\%20mais\%20comuns\%20da,das\%20m\%C3\%A3os\%20ou\%20dos\%20pés.

Organização Pan-Americana de Saúde. Organização Mundial da Saúde (2021b). Transmissão do SARS-CoV-2: implicações para as precauções de prevenção de infecção. Resumo científico 9 de julho de 2020. https://iris.paho.org/bitstream/handle/10665.2/52472/OPASWBRACOVID1920089_por.pdf?sequence=1\&isAllowed=y. 
Research, Society and Development, v. 10, n. 12, e195101220248, 2021

(CC BY 4.0) | ISSN 2525-3409 | DOI: http://dx.doi.org/10.33448/rsd-v10i12.20248

Organização Pan-Americana de Saúde. Organização Mundial da Saúde (2021c). Vacinas contra COVID-19. https://www.paho.org/pt/vacinas-contra-covid19/perguntas-frequentes-vacinas-contra-covid-19.

Pereira, A. S., Shitsuka, D. M., Parreira, F. J., \& Shitsuka, R. (2018). Metodologia da pesquisa científica. UFSM. https://repositorio.ufsm.br/bitstream/h andle/1/15824/Lic_Computacao_Metodologia-Pesquisa-Cientifica.pdf?sequence=1.

Prefeitura da Cidade do Rio de Janeiro. (2021). Informações sobre o novo Coronavírus. https://www.rio.rj.gov.br/web/transparencia/legislacao-coronavirus.

Santos, J. L. G., \& Lima, M. A. D. S. (2011). Gerenciamento do cuidado: ações dos enfermeiros em um serviço hospitalar de emergência. Rev Gaúcha Enferm. 32(4), 695-702. https://doi.org/10.1590/S1983-14472011000400009.

Sociedade Brasileira de Endoscopia Digestiva (2020). Recomendações SOBED para endoscopia segura durante a pandemia por Coronavirus. https://www.sobed.org.br/sobed-comunicacao/noticias/single/nid/recomendacoes-sobed-para-endoscopia-segura-durante-a-pandemia-por-coronavirus/.

World Health Organization. (2020a). Novel Coronavirus (2019-nCoV): Situation Report - 22. https://www.who.int/docs/default-source/coronaviruse/situationreports/20200211-sitrep-22-ncov.pdf.

World Health Organization. (2020b). Home / Health topics / Coronavirus: Symptoms. https://www.who.int/health-topics/coronavirus\#tab=tab_3.

World Health Organization. (2020c). 2019 Novel Coronavirus (2019-nCoV): Strategic preparedness and response plan. https://www.who.int/docs/defaultsource/coronaviruse/srp-04022020.pdf?ua=1.

World Health Organization (2021). Infection prevention and control during health care when coronavirus disease (COVID-19) is suspected or confirmed Interim guidance. https://www.who.int/publications/i/item/WHO-2019-nCoV-IPC-2021.1. 